\title{
The search of somatic embryogenesis regulators in Medicago truncatula
}

Tvorogova V.E.*, Baltin S.M., Krasnoperova E.Y., Potsenkovskaia E.A., Yakovleva D.V., Zlydneva N.S., Lutova L.A.

St. Petersburg State University, St. Petersburg, Russia

*email: krubaza@mail.ru

Somatic embryogenesis (SE) as a regeneration pathway is widely used in plant biotechnology for genetic modification and genome editing. The investigation of SE regulators is important for the development of new efficient protocols of plant regeneration. In recent several years, a number of morphogenic regulators were found among plant transcription factors, whose ectopic expression can stimulate SE or, in some cases, can make it possible. WUSCHEL homeodomain-containing protein from the WOX family is the most well-known example of such SE stimulator. Previously in our research, we found that ectopic expression of another gene from the WOX family, MtWOX9-1, can stimulate SE in Medicago truncatula. The aim of our present research is to find out the main factors, related to MtWOX9-1 in the regulatory network controlling SE. Phylogeny and expression analysis allowed us to find other WOX genes which can possibly act redundantly with MtWOX9-1 during SE. As far as WOX genes are known to be regulated by CLAVATA systems, we searched for CLE peptide which could suppress or activate MtWOX9-1 expression and, therefore, to suppress or stimulate SE itself. We found two possible SE regulators among CLE genes. Using transcriptome and ChIP analysis, we also search for MtWOX9-1 direct targets.

Acknowledgements: The research was made with support of the Ministry of Science and Higher Education of the Russian Federation in accordance with agreement No. 075-152020-922 date 16.11.2020 on providing a grant in the form of subsidies from the Federal budget of Russian Federation. The grant was provided for state support for the creation and development of a World-class Scientific Center "Agrotechnologies for the Future". 